misinterpreted as a lymphatic trunk. For both, although nerve correction was done during surgery by suturing, rehabilitation was necessary. For open surgery, we have never experienced obturator nerve injury during the pelvic lymphadenectomy in the same period.

Conclusions Obturator nerve injury during pelvic surgery is possibly frequent in laparoscopic surgery.

\section{EPV254/\#148 SEPTUAGENARIANS AND OCTOGENARIANS UNDERGOING GYNECOLOGIC ONCOLOGY LAPAROTOMY: IS THERE A ROLE FOR ROUTINE POSTOPERATIVE CARDIAC BIOMARKER MONITORING?}

${ }^{1} \mathrm{~T}$ Anpalagan*, ${ }^{1} \mathrm{~K}$ Huang, ${ }^{2} \mathrm{M}$ Marcucci, ${ }^{3} \mathrm{~S} J$ Mah, ${ }^{3} \mathrm{~V}$ Carlson, ${ }^{3} \mathrm{~L}$ Eiriksson, ${ }^{3} \mathrm{~W}$ Jimenez, ${ }^{3} \mathrm{C}$ Reade, ${ }^{3} \mathrm{JMV}$ Nguyen. ${ }^{1} \mathrm{McM}$ aster University, Michael G. Degroote School of Medicine, Hamilton, Canada; ${ }^{2}$ McMaster University, Juravinski Hospital and Cancer Centre, Department of Medicine, Hamilton, Canada; ${ }^{3}$ McMaster University, Juravinski Hospital and Cancer Centre, Gynecologic Oncology, Hamilton, Canada

\subsection{6/ijgc-2021-IGCS.325}

Objectives Accumulating evidence correlates myocardial injury after noncardiac surgery(MINS), even when asymptomatic, with increased cardiac and non-cardiac morbidity and mortality. There is no literature on MINS specific to Gynecologic Oncology. We sought to evaluate the incidence and risk factors of MINS in patients aged $\geq 70$.

Methods Elective laparotomies between 01/2016-09/2020 for patients aged $\geq 70$ at a tertiary hospital in ON, Canada, were reviewed using prospectively-collected National Surgical Quality Improvement Program(NSQIP) data. MINS was defined as peak serum high-sensitivity troponin- $T$ concentration $\geq 0.04 \mathrm{ng} /$ $\mathrm{mL}$ within 30 days postoperatively. Logistic regression analysis was performed.

Results In this cohort of 258 patients, of 242(93.8\%) who underwent postoperative troponin screening, 40(16.5\%) experienced MINS without exhibiting ischemic symptoms or ECG changes. The diagnosis of MINS led to a change in cardiovascular medications for 35 patients(87.5\%). On univariate analysis, Revised Cardiac Risk Index(RCRI) of 3-5 $(p=0.002)$, history of coronary artery disease $(p=0.003)$ or insulin-dependent diabetes $(\mathrm{p}=0.006)$, preoperative use of antiplatelets $(p=0.009)$, beta-blockers $(p=0.02)$, ACE-inhibitors(ACEI) or angiotensin-receptor blockers $(A R B)(p=0.020)$ and frailty as defined by the NSQIP modified frailty index$5(p=0.02)$, were associated with greater risk of MINS. Factors reflecting surgical complexity including surgical complexity score, operative duration, blood loss and advanced oncologic stage, were not predictive. Multivariable analysis using backward selection procedure identified elevated RCRI and preoperative $\mathrm{ACE} / \mathrm{ARB}$ as significant risk factors (OR5.93,95\%CI 1.52-23.31, $\mathrm{p}=0.01$ and OR2.3,95\%CI $1.18-5.06, \mathrm{p}=0.02)$.

Conclusions One in 6 patients in our cohort experienced asymptomatic MINS, irrespective of surgical complexity. MINS may be underdiagnosed after Gynecologic Oncology surgery in the absence of systematic troponin screening. Our analysis highlights a possible opportunity to optimize cardiac risk factors and potentially reduce morbidity and mortality.

\section{EPV255/\#120 TISOTUMAB VEDOTIN VS INVESTIGATOR'S CHOICE CHEMOTHERAPY IN SECOND- OR THIRD- LINE RECURRENT OR METASTATIC CERVICAL CANCER (INNOVATV 301/ENGOT-CX12/GOG-3057, TRIAL IN PROGRESS)}

${ }^{1}$ I Vergote*, ${ }^{2} \mathrm{LM}$ Randall, ${ }^{3} \mathrm{E}$ Kalbacher, ${ }^{4} \mathrm{~K}$ Madsen, ${ }^{1} \mathrm{E}$ Van Nieuwenhuysen, ${ }^{5} \mathrm{~A}$ GonzálezMartín, ${ }^{6} \mathrm{D}$ Cibula, ${ }^{7} \mathrm{~B}$ Monk, ${ }^{8} \mathrm{~L}$ Woelber, ${ }^{9} \mathrm{~S}$ Banerjee, ${ }^{10} \mathrm{~A}$ Westermann, ${ }^{11} \mathrm{~N}$ Colombo, ${ }^{12} \mathrm{D}$ Lorusso, ${ }^{13} \mathrm{P}$ Calvert, ${ }^{14} \mathrm{RL}$ Coleman, ${ }^{15} \mathrm{C}$ Marth, ${ }^{16}$ S Soumaoro, ${ }^{17} \mathrm{~S}$ Jain, ${ }^{18} \mathrm{~B}$ Slomovitz. ${ }^{1}$ Leuven Cancer Institute, Bgog and University Hospitals Leuven, Leuven, Belgium; ${ }^{2}$ Massey Cancer Center, Virginia Commonwealth University, Department of Obstetrics and Gynecology, Richmond, USA; ${ }^{3}$ Groupe d'Investigateurs Nationaux pour l'Etude des Cancers Ovariens and CHRU Jean Minjoz, Oncology, Besançon, France; ${ }^{4}$ Rigshospitalet, University Hospital of Copenhagen, and Nordic Society of Gynaecological Oncology Clinical Trial Unit (NSGO-CTU), Centre For Cancer and Organ Diseases, Copenhagen, Denmark; ${ }^{5}$ Grupo Español de Investigación en Cáncer de Ovario (GEICO) and Clínica Universidad de Navarra, Department of Medical Oncology, Madrid, Spain; ${ }^{6}$ Central and Eastern European Gynecologic Oncology Group (CEEGOG), General University Hospital, Charles University, Department of Obstetrics and Gynecology and First Faculty of Medicine, Prague, Czech Republic; ' Arizona Oncology (US Oncology Network), Gynecologic Oncology, Obstetrics and Gynecology, Phoenix, USA; ${ }^{8} A G O$ and University Medical Center Hamburg-Eppendorf, Department of Gynecology, Hamburg, Germany; ${ }^{9}$ The National Cancer Research Institute and The Royal Marsden NHS Foundation Trust, Gynaecology Unit, London, UK; ${ }^{10}$ Amsterdam University Medical Centers, Department of Medical Oncology, Amsterdam, Netherlands; " ${ }^{11}$ University of Milan-Bicocca, European Institute of Oncology, IRCCS and Mario Negri Gynecologic Oncology Group, Gynecologic Oncology Program, Milan, Italy; ${ }^{12}$ Multicentre Italian Trials in Ovarian Cancer and Gynaecological Malignancies Group (MITO) and Scientific Directorate and Department of Women and Child Health, Fondazione Policlinico Universitario Agostino Gemelli IRCCS, Gynaecology Oncology Unit, Rome, Italy; ${ }^{13}$ Cancer Trials Ireland, Gynaecology, Dublin, Ireland; ${ }^{14}$ US Oncology Research, Department of Gynecologic Oncology, The Woodlands, USA; ${ }^{15}$ Arbeitsgemeinschaft Gynäkologische Onkologie Austria (AGO-Austria), Medizinische Universität Innsbruck, Department of Obstetrics and Gynecology, Innsbruck, Austria; ${ }^{16}$ Genmab US, Inc., Oncology, Plainsboro, USA; ${ }^{17}$ Seagen Inc., Late Stage Development, Bothell, USA; ${ }^{18}$ Broward Health, Department of Gynecologic Oncology, Fort Lauderdale, USA

\subsection{6/ijgc-2021-IGCS.326}

Objectives Doublet chemotherapy (paclitaxel plus either platinum or topotecan) with bevacizumab (if eligible) is recommended for first-line treatment of recurrent/metastatic cervical cancer (r/mCC; Tewari 2014). In the second-line setting, there are limited data for available treatment options. Tisotumab vedotin (TV) is an investigational antibody-drug conjugate directed to tissue factor. In the phase 2 pivotal trial (innovaTV 204/ENGOT-cx6/GOG-3023) in r/mCC patients with disease progression on or after chemotherapy, TV demonstrated clinically meaningful and durable activity (objective response rate [ORR]: 24\%; median duration of response [DOR]: 8.3 months) with a manageable and tolerable safety profile. Most adverse events associated with TV were mild to moderate. These findings support further investigation of TV in patients with $\mathrm{r} / \mathrm{mCC}$ who progress on first-line treatment.

Methods innovaTV 301/ENGOT-cx12/GOG-3057 (NCT04697628) is a global, randomized, open-label, phase 3 trial evaluating efficacy and safety of TV in patients with previously treated $\mathrm{r} / \mathrm{mCC}$. Eligible patients are $\geq 18$ years, have $\mathrm{r} /$ mCC, and have progressed after 1-2 prior lines of therapy (either standard of care systemic chemotherapy doublet or platinum-based therapy with bevacizumab, if eligible). Approximately 482 patients will be randomized 1:1 to receive 21-day cycles of TV $(2.0 \mathrm{mg} / \mathrm{kg}$ IV once every 3 weeks $)$ or investigator's choice of chemotherapy: topotecan, vinorelbine, gemcitabine, irinotecan, or pemetrexed. The primary endpoint is overall survival. Key secondary endpoints are progression-free survival, ORR, time to response, DOR, safety, and quality of 